\title{
Comparative transcriptome analysis of scaled and scaleless skins in Gymnocypris eckloni provides insights into the molecular mechanism of scale degeneration
}

\author{
Xiu Feng ${ }^{1} \mathbb{B}$, Yintao Jia', Ren Zhu', Kemao Li², Zhongzhi Guan² and Yifeng Chen ${ }^{1 *}$
}

\begin{abstract}
Background: The scale degeneration is thought to be related to the adaptation to the extreme environment with cold climate and high-altitude in schizothoracine fishes. Gymnocypris eckloni, a schizothoracine fish living in plateau waters with the elevation above $2500 \mathrm{~m}$, is nearly esquamate and only covered with shoulder scales and anal scales, making it a good model species to study the molecular mechanism of scale degeneration.

Results: The transcriptomes of shoulder scaled skins (SSS), anal scaled skins (ASS) and scaleless skins (NSS) were sequenced and analyzed in G. eckloni at the age of 1 year. Histological examination showed that shoulder scale had completed its differentiation and anal scale just initiated the differentiation. A total of 578,046 unigenes were obtained from the transcriptomes, with 407,799 unigenes annotated in public databases. A total of 428 and 142 differentially expressed unigenes (DEUs) were identified between SSS and NSS, and between ASS and NSS, respectively, with 45 DEUs that were overlapped. Annotation analysis indicated that these DEUs were mainly enriched in Gene Ontology (GO) terms and KEGG pathways associated with bone and muscle formation, such as myofibril, contractile fiber, cytoskeletal protein binding, muscle structure development, cardiac muscle contraction, hypertrophic cardiomyopathy (HCM) and calcium signaling pathway.
\end{abstract}

Conclusions: Our results would provide insights into the molecular mechanisms of scale degeneration in G. eckloni and other congeneric fishes. In addition, the transcriptome data provides candidate genes and markers for future studies.

Keywords: Gymnocypris eckloni, Transcriptome, Skin, Scale degeneration

\section{Background}

The scales in fish species refer to the dermis-derived structures located within the skin, and are classified into placoid, ganoid and elasmoid (cycloid and ctenoid) [1-3]. Most scales in teleosts belong to the elasmoid type, which is a highly derived type of scale [1]. Many studies have described the scale formation process at the tissue and cellular level [1, 4-7], but little is known at the molecular level. Only several candidate

\footnotetext{
* Correspondence: chenyf@ihb.ac.cn

${ }^{1}$ Institute of Hydrobiology, Chinese Academy of Sciences, Wuhan 430072, China

Full list of author information is available at the end of the article
}

genes are supposed to contribute the fish scale formation. In zebrafish (Danio rerio), whole-mount in situ hybridization revealed that sonic hedgehog (shh) may be involved in the control of scale morphogenesis and differentiation [1]. In medaka (Oryzias latipes), a mutation at the rs-3 locus encoding ectodysplasin-A receptor (EDAR) leads to almost complete loss of scales, indicating that EDAR is required for scale development $[8,9]$. Based on phylogenetic analyses, ectodysplasin- $A$ (Eda) and secretory calcium-binding phosphoproteins (SCCPS) are also supposed to be associated with the scale development in fish species [10-12]. However, very few studies have been conducted using genomic or transcriptomic analyses. 
The transcriptome represents the set of all transcripts expressed in one cell or a population of cells. With the development and popularization of the next-generation sequencing technologies (NGS), whole transcriptome sequencing or RNA sequencing (RNA-seq) has been widely used for transcriptome analysis at massive scale [13]. Comparative transcriptome analysis provides a powerful tool for dissecting the relationship between genotype and phenotype, increasing our understanding of the molecular mechanisms involved in physiological process and environmental adaptation [14]. For example, comparative transcriptome analysis by RNA-seq has identified genes and pathways associated with growth [15], gonad development [16], immune response [17] and skin color differentiation [18] in fish species.

The schizothoracine fishes (Teleostei: Cyprinidae) are mainly distributed in the Qinghai Tibetan Plateau (QTP) and its surrounding areas, and are the largest group of the QTP ichthyofauna [19]. Among them, Gymnocypris fishes mainly live in the cold and high altitude area on the QTP, which are nearly esquamate, only covered with 3-4 rows of shoulder scale, and two lines of anal scale [19, 20]. The degenerated scale and incrassated skin are two characteristics of Gymnocypris fishes which related to the adaptation to the extreme environment with cold climate and highaltitude. Uncovering the molecular basis that controlling the scale degeneration would provide new insights into how the Gymnocypris fishes adapt to the extreme environment of the QTP. The presence of shoulder scales and anal scales indicate that the scale degeneration may be involved in gene expression regulation rather than the loss of a single or a few genes. Previous studies of Gymnocypris fishes have made great progress in phylogenetics, biogeography and ecology [21-24]. However, the molecular mechanism of scale degeneration is still not studied.

Gymnocypris eckloni, a representative species belonging to the genus Gymnocypris, is a native fish species in the upper reaches of the Yellow River with the elevation above $2500 \mathrm{~m}$. In this study, we sequenced and analyzed the transcriptomes of scaled and scaleless skin tissues in G. eckloni. The aim of this study is de novo assembly of the transcriptome and identification of differentially expressed unigenes that may be involved in scale degeneration in G. eckloni.

\section{Results}

\section{Histology observation of skin tissues}

At age of 1 year, juvenile specimens of G. eckloni (with the average body weight and body length of $15.01 \mathrm{~g}$ and 10.23 $\mathrm{cm}$ ) showed obvious scales on the shoulder skin but only slight folds on the anal skin (Fig. 1). Histological examination showed that the shoulder scale had completed its differentiation, and its posterior region had protruded into the epidermis which formed a fold. The anal scale had just reached at the early stage of development, the scale papilla had differentiated in the dermis. The scaleless skin showed no morphogenesis of scale development.

\section{Transcriptome sequencing, assembly and annotation}

The transcriptomes of shoulder scaled skins (SSS), anal scaled skins (ASS) and scaleless skins (NSS) from three fish individuals were sequenced by using Illumina paired-end sequencing technology. In total, 505.99 million raw reads were generated from all nine tissue samples (Table 1). After

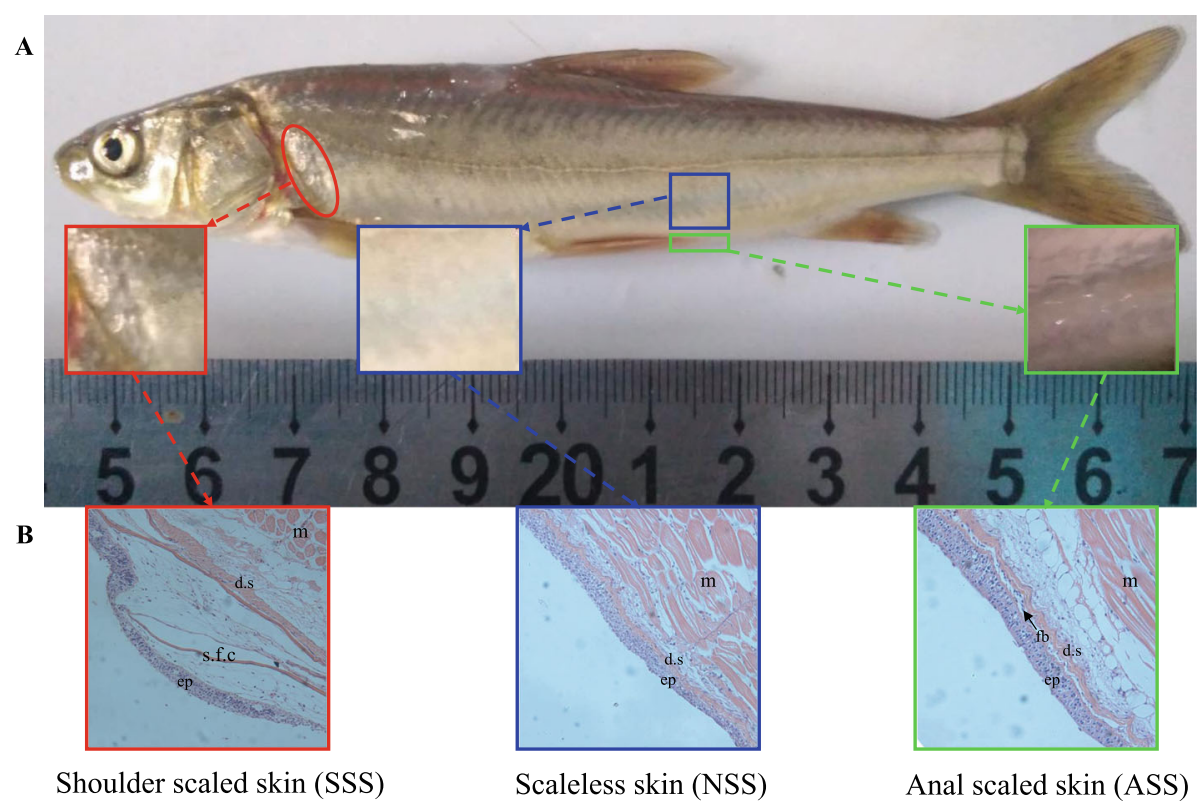

Fig. 1 The diagrammatic drawing of sample positions (a) and histology observation (magnification $\times 200$ ) (b) of skin tissues in G. eckloni. ep, epidermis; d.s, dermal stroma; m, muscle; s.f.c, scale-forming cells; fb: fibroblast 
Table 1 Summary for the transcriptome of G. eckloni using Illumina RNA-seq

\begin{tabular}{ll}
\hline Prameters & Data \\
\hline Number of raw reads & $505,993,248$ \\
Number of clean reads & $493,470,036$ \\
Number of unigenes & 578,046 \\
Mean length of unigenes (bp) & 747 \\
N50 length of unigenes (bp) & 1193 \\
\hline
\end{tabular}

trimming and filtering, a total of 493.47 million clean reads were obtained, with the average number of reads of 54.83 million for each tissue sample (Additional file 1). A total of 771,455 transcripts from 433,844 'genes' were de novo assembled by Trinity software [25]. After clustering by CDHIT-EST [26], 578,046 unigenes were obtained with the average length and N50 length of $747 \mathrm{bp}$ and $1193 \mathrm{bp}$.

The unigenes were annotated based on the public databases. A total of 490,581, 111,659, 70,059, 66,746, 68,274 and 30,004 unigenes were assigned to NCBI non-redundant nucleotide sequences $(\mathrm{Nt})$, non-redundant protein sequences (Nr), Swissprot, Cluster of Orthologous Groups of proteins (KOG), Gene Ontology (GO) and KEGG Ortholog database (KEGG), respectively, with 25,799 unigenes annotated in all databases and 407,799 unigenes annotated in at least one database (Fig. 2). GO annotations generated 66 level 2 GO terms under three functional categories: cellular component, molecular function and biological process (Additional file 2). For KEGG annotation, the 'signal transduction' pathway had the largest number of unigenes $(n=$ $2672)$, followed by 'global and overview maps' $(n=2121)$ and 'infectious disease: viral' $(n=1422)$ (Additional file 3). Similar results of GO and KEGG annotations have also been reported in a previous study [27].
Identification of differentially expressed unigenes (DEUs) Based on the criteria of $\left|\log _{2} \mathrm{FC}\right| \geq 2$ and $p$-value $\leq 0.001$, a total of 428 and 142 DEUs were identified between SSS and NSS, and between ASS and NSS, respectively. Compared with NSS, SSS had 75 up-regulated and 353 down-regulated unigenes, and ASS had 39 up-regulated and 103 down-regulated unigenes. The Venn diagram showed that 17 and 28 unigenes were up-regulated and down-regulated, respectively, in both SSS and ASS compared with NSS (Fig. 3). Based on the global expression profiles, the samples were clustered into three groups corresponding to the three fish individuals (Fig. 4a). However, based on the expressions of DEUs, the samples were clustered into two groups representing scaleless and scaled skins (Fig. 4b).

\section{Functional annotation of DEUs}

A total of 384, 349 and 207 DEUs for SSS, and 127, 110 and 58 DEUs for ASS were assigned to $\mathrm{Nr}, \mathrm{GO}$ and KEGG databases, respectively (Additional file 4). For overlapped DEUs, 38, 33 and 19 unigenes were annotated to the three databases.

GO enrichment analysis showed that DEUs for SSS were mainly enriched in 'myofibril' (GO:0030016), 'contractile fiber' (GO:0043292) and 'actin cytoskeleton' (GO:0015629) under cellular component, 'actin binding' (GO:0003779), 'actin filament binding' (GO:0051015) and 'cytoskeletal protein binding' (GO:0008092) under molecular function, and 'muscle structure development' (GO:0061061), 'muscle cell development' (GO:0055002) and 'myofibril assembly' (GO:0030239) under biological process (Table 2). DEUs for ASS had similar enriched terms under cellular component, with much lower adjusted $p$-values compared with DEUs for SSS.

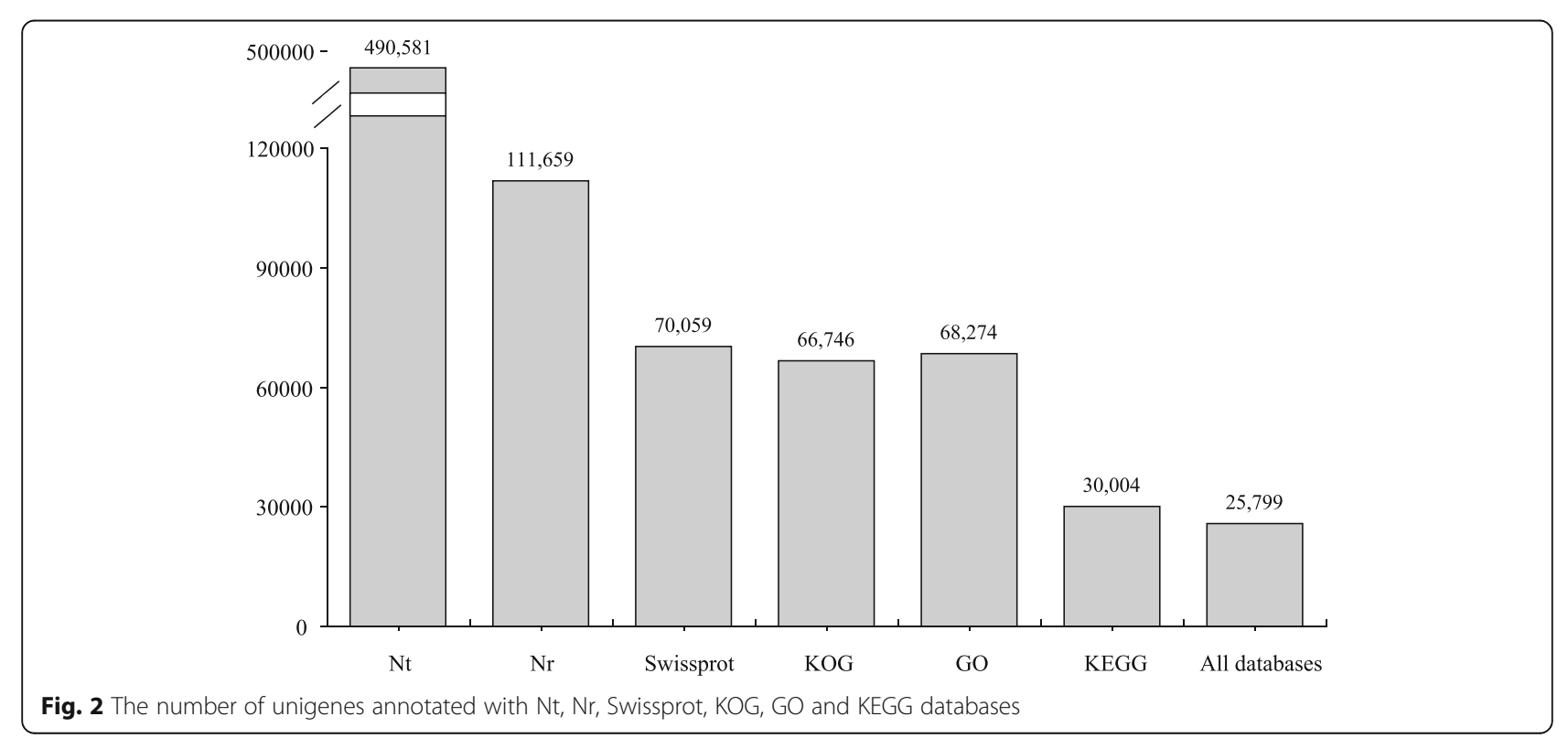




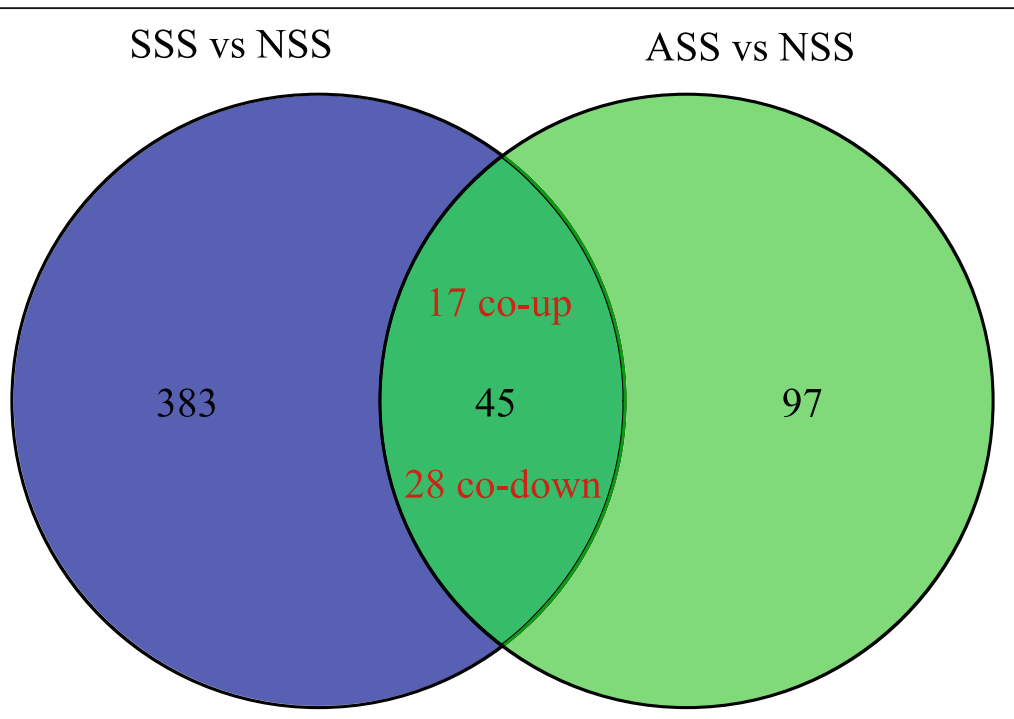

Fig. 3 The venn diagram of differentially expressed unigenes (DEUs). SSS, shoulder scaled skins; ASS, anal scaled skins; NSS, scaleless skins

KEGG enrichment analysis showed that DEUs for SSS were mainly enriched the pathways 'cardiac muscle contraction', 'hypertrophic cardiomyopathy (HCM)', 'dilated cardiomyopathy' and 'calcium signaling pathway' (Fig. 5a). Among the top 20 of enriched pathways, six were enriched for both DEUs for SSS and DEUs for ASS (Fig. $5 b)$. However, DEUs for SSS had much higher enrichment scores than DEUs for ASS.

The overlapped DEUs only contain 45 unigenes (Table 3). The top five up-regulated unigenes (according to $\log _{2} \mathrm{FC}$ ) were UPF0575 protein C19orf67 homolog, CUB and zona pellucida-like domain-containing protein
1 , sodium/calcium exchanger 1 , osteocalcin and galectin4. The top five down-regulated unigenes include melanotransferrin, tensin, myozenin-2, E3 ubiquitin-protein ligase TRIM21 and troponin I, slow skeletal muscle.

\section{Verification of gene expression}

To assess the reliability of RNA-seq quantification analysis, ten DEUs were randomly selected for analysis using real-time reverse transcription quantitative PCR (qRT-PCR). The $\beta$-actin (DN589_c0_g1_i2) was used for the normalization in $\mathrm{qRT}-\mathrm{PCR}$, as its expression levels were confirmed to be consistent among the three groups
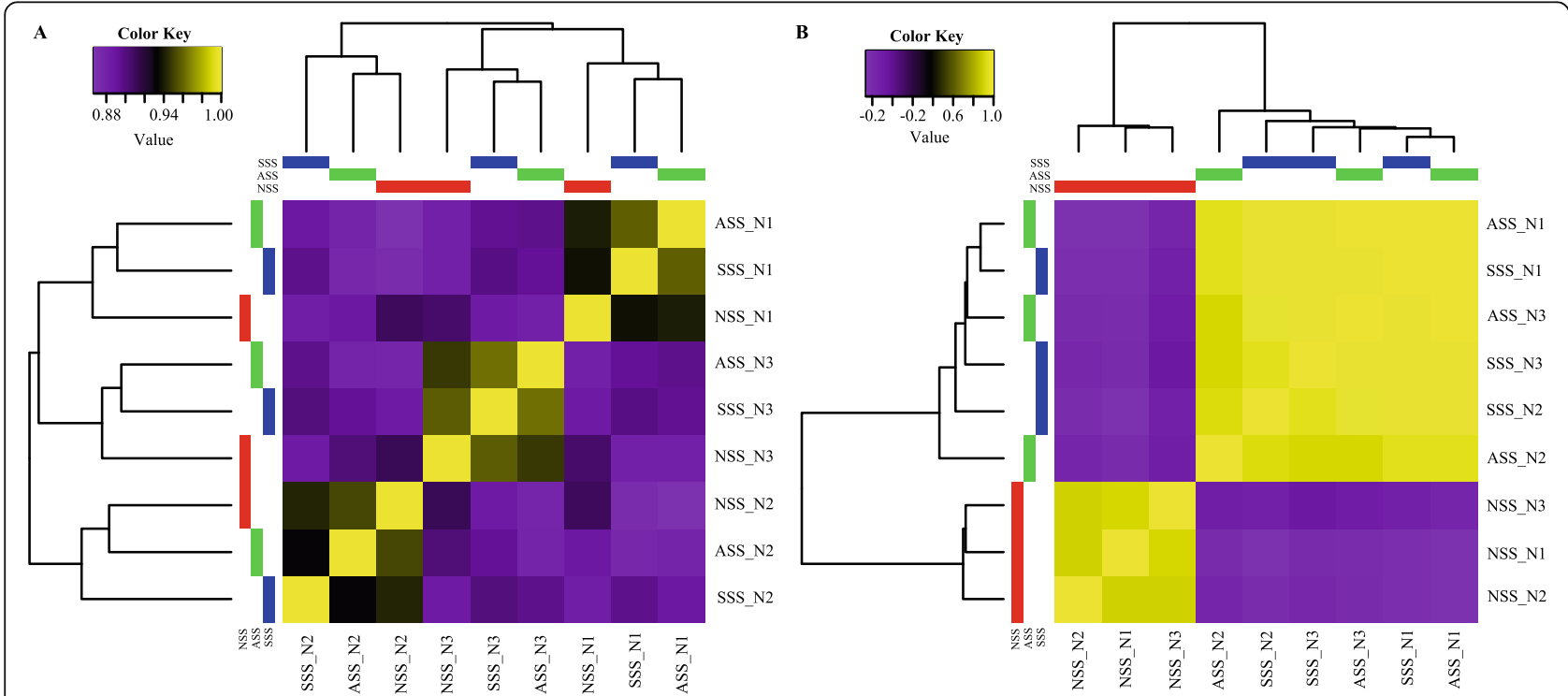

Fig. 4 Heat map showing the pairwise Spearman correlations among various tissues based on expression profiles of all unigenes (a) and DEUs (b) 
Table 2 GO enrichment analysis of DEUs

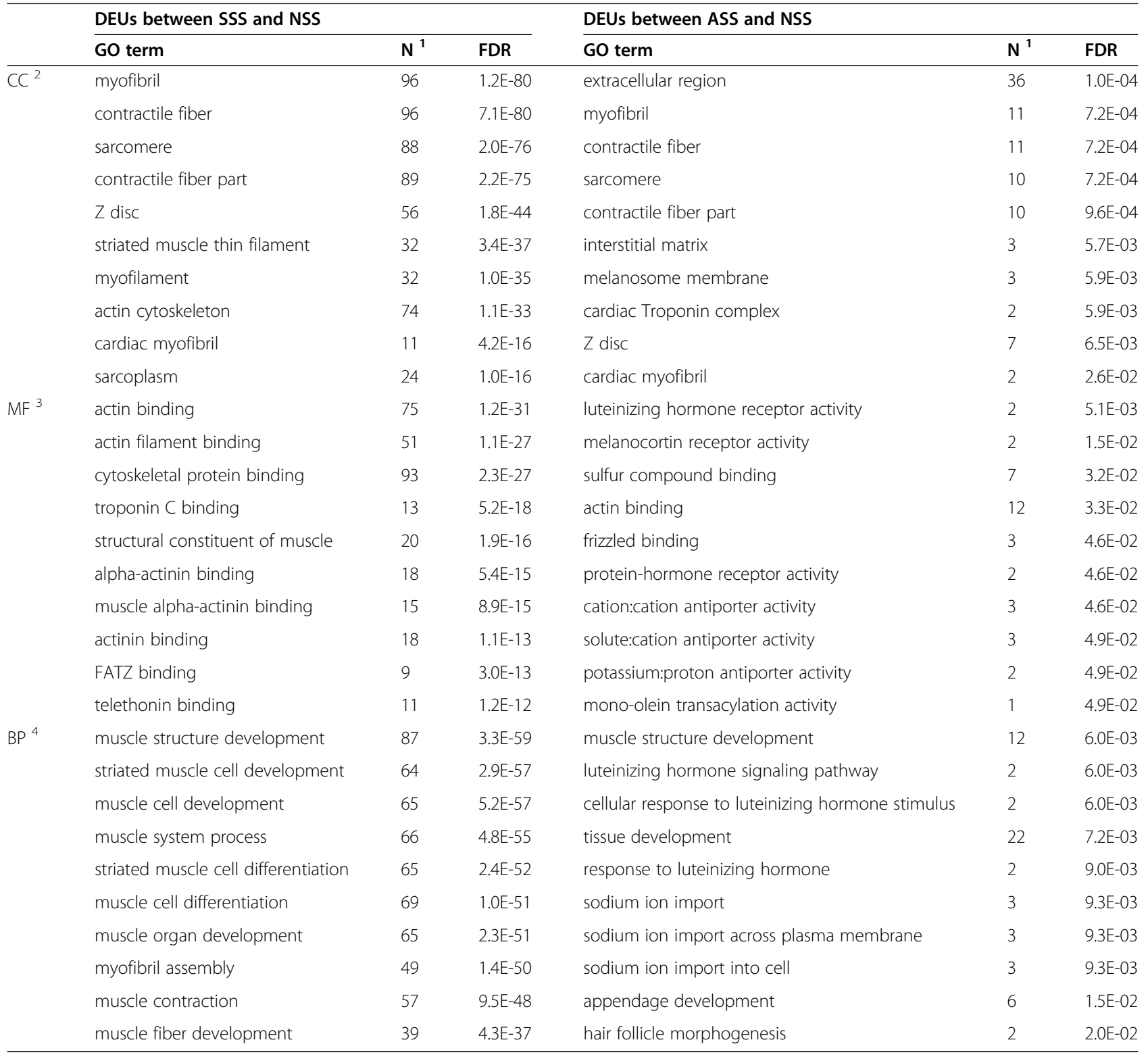

based on RNA-seq data. The results showed that the expressions of the selected DEUs were all validated by qRT-PCR (Fig. 6). The Spearman correlation coefficient between the relative expression levels obtained by qRTPCR and FPKM values and was $0.92(p<0.01)$.

\section{Discussion}

Fish scales are derived from the dermis located within the skin [1]. The positions of the first appearance of scales vary among different fish species. In this study, the scales on the shoulder and anal of G. eckloni were observed, and the transcriptomes of their derived skins were sequenced and analyzed. The scales appeared first on the shoulder, which was similar to other cyprinid fishes but different from cichlid and catostomid fishes. The first scale generally appears in the anterior region of the body in cyprinid fishes $[5,28]$, and in the posterior region of the body in cichlid and catostomid fishes $[29,30]$. The difference of scale development process may be related to species evolution and environmental adaptation.

Very large number of unigenes $(578,046)$ was obtained from the assembled transcriptome in this study, which is similar to that previously reported in the same species $(551,430)$ [27] as well as in another congeneric species $(532,241)$ [24]. The main reason for this phenomenon may be that Gymnocypris fishes are tetraploid with large numbers of chromosomes and big genome sizes [31, 32]. Based on global expression profiles, tissue samples were 


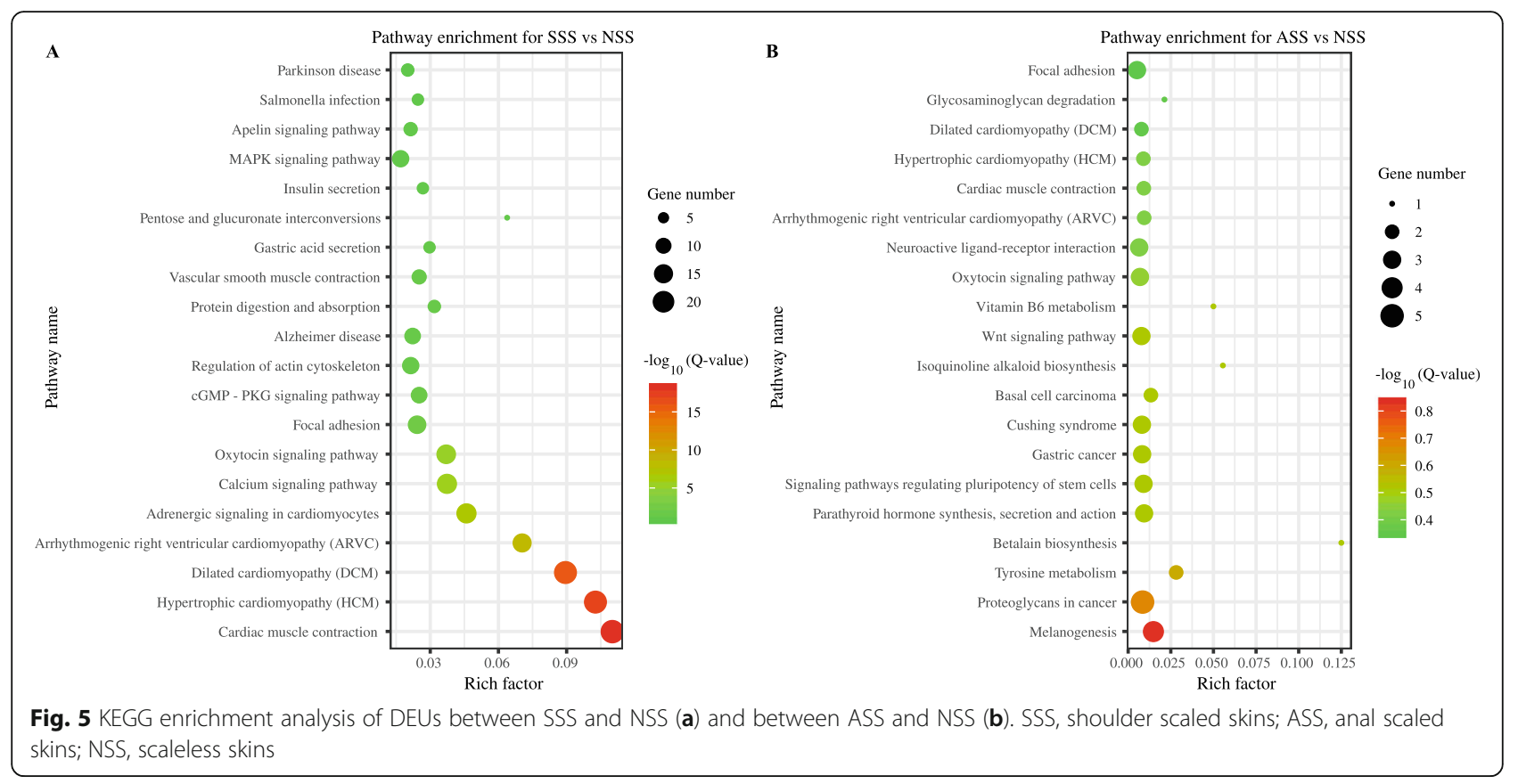

clustered into groups representing fish individuals and not the skin types. An explanation may be that the scales are derived from skins, and the difference in expression between scaled and scaleless skins was smaller than that between individuals. At age of 1 year, shoulder scale completed its differentiation and anal scale just initiated the differentiation. More DEUs were detected between SSS and NSS (428) than between ASS and NSS (142), indicating that more and more genes may be involved in the process of scale development.

DEUs between scaled and scaleless skins were mainly enriched in GO terms associated with bone and muscle formation, such as 'myofibril', 'contractile fiber', 'cytoskeletal protein binding', and 'muscle structure development'. These GO terms have also been reported in other studies with their functions on the development and differentiation in skin, bone and muscle [33-37]. One of the most significant up-regulated unigenes, Osteocalcin, is a marker of mature osteoblasts in mammals, which may be an important gene for scale formation [38, 39]. Osteocalcin is the most abundant noncollagenous bone protein of many fishes, such as common carp, grass carp and tilapia, but except for some scaleless fishes, such as channel catfish [40]. The fish scale is a type of dermal skeleton, so the genes related to scale formation in this study also are involved in bone formation [41]. The dermal bone of fish scales has been used as a model for bone research [42].

Previous studies have indicated that several genes may be involved in fish scale formation, such as ectodysplasin-A receptor (EDAR) [9], ectodysplasin-A (Eda) [12] and secretory calcium-binding phosphoproteins (SCPPs)
[10]. In this study, these genes were all present and expressed in both scaled and scaleless skins of G. eckloni with no significant difference. Similarly in common carp, both $E d a$ and $E D A R$ were not differently expressed during scale regeneration [10]. Furthermore, the two genes were also both present and expressed in the skin of channel catfish, a scaleless fish [10]. These findings indicate that the expression of Eda and EDAR is present in both the scaled skin and the scaleless skin, and is not the only requirement for scale development in fish species. G. eckloni, a fish species belonging to Cyprinidae, is not entirely scaleless, and is covered with shoulder scales and anal scales. The scale degeneration in this fish are not caused by the loss of key genes reported previously, and may be related to the expression regulation of genes identified in this study. Such regulation mechanism was developed to adapt to the extreme environment of the QTP, and may be mediated by transcriptional or posttranscriptional factors, such as methylation, transcription factors (TF) and microRNAs (miRNAs).

\section{Conclusions}

In summary, the transcriptomes of scaled and scaleless skins were firstly sequenced and compared in G. eckloni. The reference transcriptome with 578,046 unigenes was de novo assembled. A number of differentially expressed unigenes were identified between scaled and scaleless skins. These unigenes were mainly involved in GO terms and KEGG pathways associated with bone and muscle formation. Our results would provide insights into the molecular mechanisms of scale degeneration in G. eckloni and other congeneric fishes. 
Table 3 The overlapped DEUs between SSS vs NSS and ASS vs NSS

\begin{tabular}{|c|c|c|c|}
\hline Unigenes & Log $_{2}$ FC (SSS vs NSS) & $\log _{2}$ FC (ASS vs NSS) & Description \\
\hline DN2099_c0_g1_i7 & -11.30 & -11.21 & Melanotransferrin \\
\hline DN16257_c0_g1_i4 & -10.16 & -10.07 & Tensin \\
\hline DN10934_c0_g1_i2 & -9.80 & -9.70 & Myozenin-2 \\
\hline DN5729_c1_g1_i2 & -9.78 & -9.60 & E3 ubiquitin-protein ligase TRIM21 \\
\hline DN6176_c0_g2_i4 & -9.75 & -6.40 & Troponin I, slow skeletal muscle \\
\hline DN6176_c0_g3_i1 & -9.67 & -9.57 & Troponin I, cardiac muscle \\
\hline DN10193_c0_g2_i1 & -9.42 & -9.33 & Filamin-C \\
\hline DN3099_c0_g1_i1 & -9.26 & -9.16 & Myosin-16 \\
\hline DN6176_c0_g1_i16 & -9.00 & -8.91 & Sodium/hydrogen exchanger 7 \\
\hline DN20169_c0_g1_i10 & -8.98 & -8.89 & Ethanolamine-phosphate phospho-lyase \\
\hline DN13779_c0_g1_i7 & -8.37 & -8.27 & Voltage-dependent calcium channel subunit alpha- $2 /$ delta- 2 \\
\hline DN25484_c0_g1_i7 & -8.23 & -8.13 & Integrin alpha-7 \\
\hline DN5729_c1_g1_i11 & -7.81 & -9.58 & E3 ubiquitin-protein ligase TRIM39-like \\
\hline DN20918_c1_g1_i10 & -7.29 & -7.13 & Myosin-7 \\
\hline DN2286_c0_g1_i5 & -7.21 & -9.80 & LIM domain-binding protein 3 \\
\hline DN15718_c1_g1_i7 & -6.58 & -6.41 & Triadin-like isoform $X 4$ \\
\hline DN11289_c0_g2_i4 & -5.69 & -8.28 & Neurofilament heavy polypeptide \\
\hline DN2232_c0_g1_i16 & -4.50 & -6.35 & Elongation factor 2 \\
\hline DN45284_c0_g1_i3 & -4.36 & -4.05 & Growth/differentiation factor 8 \\
\hline DN5543_c0_g1_i16 & -2.20 & -3.38 & Fibrinogen-like protein 1 \\
\hline DN19919_c0_g1_i2 & 2.50 & 2.55 & Sclerostin \\
\hline DN15430_c0_g1_i3 & 2.87 & 2.47 & Homeobox protein Dlx5a \\
\hline DN2890_c0_g1_i11 & 3.03 & 2.87 & Pannexin-3 \\
\hline DN4077_C0_g1_i9 & 3.51 & 2.82 & DNA-binding protein SATB2 \\
\hline DN78_c0_g4_i1 & 3.61 & 2.40 & Lysyl oxidase homolog 4 \\
\hline DN8724_c0_g1_i1 & 3.87 & 3.23 & Hormonally up-regulated neu tumor-associated kinase homolog A \\
\hline DN1195_c0_g2_i1 & 3.94 & 2.51 & Collagen alpha-2(V) chain-like \\
\hline DN5111__c0_g1_i8 & 4.23 & 2.64 & Collagen alpha-1(V) chain \\
\hline DN13869_c0_g1_i1 & 5.69 & 5.01 & Mucin-5 AC \\
\hline DN10512_c0_g1_i6 & 6.87 & 7.48 & Transient receptor potential cation channel, subfamily M \\
\hline DN8192_C0_g1_i1 & 7.29 & 7.78 & Galectin-4 \\
\hline DN142241_C0_g1_i6 & 7.81 & 5.62 & Osteocalcin \\
\hline DN12086_c0_g1_i2 & 8.85 & 8.17 & Sodium/calcium exchanger 1 \\
\hline DN8352_c0_g1_i3 & 9.07 & 6.91 & CUB and zona pellucida-like domain-containing protein 1 \\
\hline DN34990_c0_g1_i10 & 11.26 & 11.71 & UPF0575 protein C19orf67 homolog \\
\hline
\end{tabular}

\section{Methods}

\section{Fish materials}

The parent population of G. eckloni used for artificial propagation and releasing was raised at the Suzhi station of fish propagation (Haidong, China). A progeny population were generated by artificial propagation in May 2018. The progenies was then raised in a 0.3 ha muddy pond and fed three times daily at QingHai Provincial Fishery Environmental Monitoring Center (Xining, China). After 1 year of culture, the neonatal shoulder scales and anal scales could be observed by optical microscope. Three fish individuals were collected after they were euthanized with an overdose of MS222 $(100 \mathrm{mg} / \mathrm{L})$. Skin tissues of shoulder scales, anal scales and no scales were sampled from each individual. One part of them was fixed in $4 \%$ paraformaldehyde, sectioned $(6 \mathrm{~mm})$ and stained by standard hematoxylin-eosin (H\&E) staining to examine the stages of scale development. Another part was immediately frozen in liquid nitrogen. 


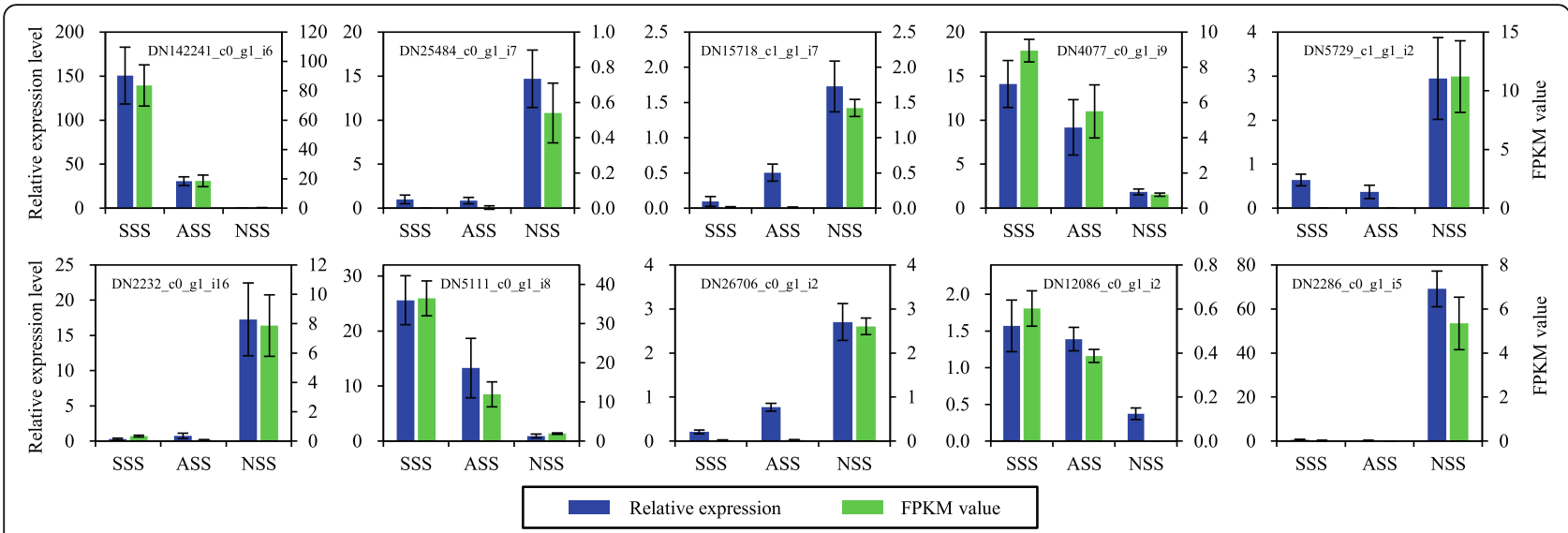

Fig. 6 Validation of the RNA-seq expression profile by real-time reverse transcription quantitative PCR. Data were shown as mean \pm SE $(n=3)$. SSS, shoulder scaled skins; ASS, anal scaled skins; NSS, scaleless skins

RNA extraction, library construction and sequencing

Total RNA was extracted using Trizol reagent (Invitrogen, Carlsbad, CA, USA) according to the manufacturer's instructions. RNA degradation and contamination were assessed by ethidium bromide staining of $28 \mathrm{~s}$ and $18 \mathrm{~s}$ ribosomal RNA on a $1 \%$ agarose gel [43]. The RNA integrity was then checked using an Agilent 2100 Bioanalyzer (Agilent Technologies, Palo Alto, CA, USA) with the cutoff value of RNA Integrity Number (RIN) $\geq 7$. The RNA concentration was measured using a Qubit RNA HS Assay Kit in Qubit 2.0 Flurometer (Life Technologies, Carlsbad, CA, USA). The RNA-seq libraries were constructed using the TruSeq RNA Sample Prep Kit (Illumina, San Diego, CA, USA) following the manufacturer's instructions. Briefly, mRNA was enriched using magnetic beads with Oligo (dT) and fragmented using divalent cations at elevated temperature. The RNA fragments were reverse transcribed into first strand CDNA with a six-base random primer, followed by second-strand cDNA synthesis, 3' end repair and ligation of adapters. The ligated fragments were enriched by PCR to generate the final cDNA library. Finally, nine libraries (3 replicates for each kind of skin tissue) were constructed and sequenced on an Illumina HiSeq X Ten sequencer to generate $150 \mathrm{bp}$ pair-end $(\mathrm{PE})$ reads.

\section{De novo assembly and annotation}

The raw paired-end reads were filtered to obtain highquality clean reads using fastp 0.18 .0 with the following parameters: -q 28 -u 20 -1 50-3 -W 4 -M 30 [44]. Owing to the absence of a reference genome of G. eckloni, the transcriptome was de novo assembled using Trinity 2.8.4 software based on merged clean paired-end reads [25]. The assembled unigenes were obtained after a clustering with CD-HIT-EST $(c=0.95)$ [26]. The unigenes were annotated by searching against six databases with the latest releases includingNr, Nt, COG, Swissprot, GO and KEGG. Three softwares were used for functional annotation with the evalue of $1 \mathrm{e}-10$, including BLAST + for $\mathrm{Nt}$ [45], Diamond for Nr, KOG, Swissprot and KEGG [46, 47], and Blast2GO for $\mathrm{GO}$ [48].

\section{Analysis of differentially expressed unigenes}

Clean reads of each RNA-seq library were aligned to the assembled reference transcriptome to obtain unique mapped reads by using bowtie 2 software [49]. The expression level of each unigene for each sample was calculated and normalized into FPKM (reads per kilobase of a gene per million reads) values by RSEM software [50]. All samples were divided into three groups, including SSS (shoulder scaled skin tissues), ASS (anal scaled skin tissues) and NSS (scaleless skin tissues). The count data were used to identify the differentially expressed unigenes between the group SSS and NSS, and ASS and NSS using the R package edgeR [51]. To limit false positive findings, unigenes with $\left|\log _{2} \mathrm{FC}\right| \geq 2$ and $p$-value $\leq 0.001$ were identified as DEUs.

\section{GO and KEGG enrichment analysis of DEUs}

To further understand the DEUs' biological functions, GO and KEGG enrichment analysis were performed using the hypergeometric Fisher exact test in an online tool (OmicShare, www.omicshare.com/tools). The whole transcriptome was set as the background. The enriched GO and KEGG terms were confirmed with a threshold of false discovery rate (FDR)-corrected $p$-value $<0.05$.

\section{qRT-PCR validation of DEUs}

Ten DEUs were randomly selected for validation using qRT-PCR. The RNA samples used for RNA-seq library construction were also used for qRT-PCR. The firststrand cDNA was synthesized from $1 \mu \mathrm{g}$ RNA using MMLV Reverse Transcriptase (TaKaRa, Japan) with oligo (dT) primer. The qRT-PCR reactions were carried out 
on a Bio-Rad CFX-96 real-time PCR system (Bio-Rad, Hercules, USA) with the SYBR Premix ExTaq ${ }^{\text {TM }}$ Kit (Takara, Japan). The relative expression levels were normalized to the quantification of $\beta$-actin using the $2^{-}$ $\triangle \Delta C T$ method [52].

\section{Supplementary Information}

The online version contains supplementary material available at https://doi. org/10.1186/s12864-020-07247-w.

Additional file 1: Table S1. Summary of the sequencing data per sample in this study.

Additional file 2: Figure S1. Gene Ontology (GO) classification of all unigenes.

Additional file 3: Figure S2. KEGG classification of all unigenes.

Additional file 4: Table S2. Annotation of differentially expressed unigenes (DEUs) between NSS and others.

\section{Abbreviations}

DEUs: Differentially expressed unigenes; Eda: Ectodysplasin-A; EDAR: Ectodysplasin-A receptor; GO: Gene ontology; KEGG: Kyoto encyclopedia of genes and genomes; COGs: Cluster of orthologous groups of proteins; NGS: Next-generation sequencing; qRT-PCR: Reverse transcription quantitative PCR; QTP: Qinghai Tibetan Plateau; RNA-seq: RNA sequencing; SCCPs: Secretory calcium-binding phosphoproteins; Shh: Sonic hedgehog; TF: Transcription factors

\section{Acknowledgements}

We thank Dr. Dekui He and Dr. Xiaoyun Sui for their assistance in field and laboratory work.

\section{Authors' contributions}

XF and YC conceived and designed the study. XF, YJ and RZ conducted the experiment. $\mathrm{KL}$ and $Z \mathrm{G}$ contributed sample collection and new reagents. $\mathrm{XF}$ performed the data analysis and wrote the manuscript. All authors read and approved the final manuscript.

\section{Funding}

This research was supported by the Second Tibetan Plateau Scientific Expedition and Research Program (STEP, Grant No. 2019QZKK0304), the Strategic Priority Research Program of the Chinese Academy of Sciences (Grant No. XDB31040101), the National Natural Science Foundation of China (Grant No. 31601844), and the Knowledge Innovation Program of the Chinese Academy of Sciences (Grant No. Y85E05). These funding bodies had no role in the design of the study and collection, analysis, and interpretation of data and in writing the manuscript.

\section{Availability of data and materials}

All raw transcriptome data reported in this article have been deposited in the Genome Sequence Archive (GSA; http://gsa.big.ac.cn/) under accession number PRJCA003014.

\section{Ethics approval and consent to participate}

All experimental procedures were approved by the Animal Care and Use Committee at the Institute of Hydrobiology, Chinese Academy of Sciences.

\section{Consent for publication}

Not applicable.

\section{Competing interests}

The authors declare that they have no competing interests.

\section{Author details}

${ }^{1}$ Institute of Hydrobiology, Chinese Academy of Sciences, Wuhan 430072, China. ${ }^{2}$ Qing Hai Provincial Fishery Environmental Monitoring Center, Xining 810012 , China.
Received: 9 July 2020 Accepted: 18 November 2020

Published online: 27 November 2020

\section{References}

1. Sire JY, Akimenko MA. Scale development in fish: a review, with description of sonic hedgehog (shh) expression in the zebrafish (Danio rerio). Int J Dev Biol. 2003:48:233-47.

2. Zhu D, Ortega CF, Motamedi R, Szewciw L, Vernerey F, Barthelat F. Structure and mechanical performance of a "modern" fish scale. Adv Eng Mater. 2012; 14:B185-94

3. Yang W, Gludovatz B, Zimmermann EA, Bale HA, Ritchie RO, Meyers MA Structure and fracture resistance of alligator gar (Atractosteus spatula) armored fish scales. Acta Biomater. 2013:9:5876-89.

4. Franklin DR, Smith LL Jr. Note on development of scale patterns in the northern pike, Esox lucius L. T. Am Fish Soc. 1960;89:83.

5. McCrimmon HR, Swee UB. Scale formation as related to growth and development of young carp, Cyprinus carpio L. J Fish Res Bd Can. 1967;24:47-51

6. Sire JY, Quilhac A, Bourguignon J, Allizard F. Evidence for participation of the epidermis in the deposition of superficial layer of scales in zebrafish (Danio rerio): a SEM and TEM study. J Morphol. 1997:231:161-74.

7. Yan TM, Tang RJ, Liu XS, Yang SY, Yang S, He Z. The scale formation and development in juvenile of Schizothorax prenanti. Acta Hydrob Sin. 2014;38: 298-303.

8. Sharpe PT. Fish scale development: hair today, teeth and scales yesterday? Curr Biol. 2001;11:R751-2.

9. Kondo S, Kuwahara Y, Kondo M, Naruse K, Mitani H, Wakamatsu Y, Ozato K, Asakawa S, Shimizu N, Shima A. The medaka rs-3 locus required for scale development encodes ectodysplasin-a receptor. Curr Biol. 2001;11:1202-6.

10. Liu Z, Liu S, Yao J, Bao L, Zhang J, Li Y, Jiang C, Sun L, Wang R, Zhang Y, et al. The channel catfish genome sequence provides insights into the evolution of scale formation in teleosts. Nat Commun. 2016;7:11757.

11. LV Y, Kawasaki K, Li J, Li Y, Bian C, Huang Y, You X, Shi Q. A genomic survey of SCPP family genes in fishes provides novel insights into the evolution of fish scales. Int J Mol Sci. 2017;18:2432.

12. Zhang C, Tong C, Ludwig A, Tang Y, Liu S, Zhang R, Feng C, Li G, Peng Z Zhao K. Adaptive evolution of the Eda gene and scales loss in schizothoracine fishes in response to uplift of the Tibetan plateau. Int J Mol Sci. 2018;19:2953.

13. Qian X, Ba Y, Zhuang Q, Zhong G. RNA-Seq technology and its application in fish transcriptomics. OMICS. 2014;18:98-110.

14. Wang Z, Gerstein M, Snyder M. RNA-Seq: a revolutionary tool for transcriptomics. Nat Rev Genet. 2009;10:57.

15. Fu B, Wang X, Feng X, Yu X, Tong J. Comparative transcriptomic analyses of two bighead carp (Hypophthalmichthys nobilis) groups with different growth rates. Comp Biochem Physiol D. 2016;20:111-7.

16. Bar I, Cummins S, Elizur A. Transcriptome analysis reveals differentially expressed genes associated with germ cell and gonad development in the southern bluefin tuna (Thunnus maccoyii). BMC Genomics. 2016;17:217.

17. Zhang X, Wang S, Chen S, Chen Y, Liu Y, Shao C, Wang Q, Lu Y, Gong G, Ding $S$, et al. Transcriptome analysis revealed changes of multiple genes involved in immunity in Cynoglossus semilaevis during Vibrio anguillarum infection. Fish Shellfish Immunol. 2015:43:209-18.

18. Zhu W, Wang L, Dong Z, Chen X, Song F, Liu N, Yang H, Fu J. Comparative transcriptome analysis identifies candidate genes related to skin color differentiation in red tilapia. Sci Rep. 2016;6:31347.

19. Chen YF, Cao WX. Schizothoracinae. In: Yue P, editor. Fauna Sinica, Osteichthyes, Cypriniformes III. Beijing: Science Press; 2000. p. 273-335.

20. Cao WX, Chen YY, Wu YF, Zhu SQ. Origin and evolution of schizothoracine fishes in relation to the upheaval of the Xizang Plateau. In: The team of the comprehensive scientific expedition to the Qinghai-Xizang Plateau, Chinese Academy of Sciences, editor. Studies on the period, amplitude and type of the uplift of the Qinghai-Xizang Plateau. Beijing: Science Press; 1981. p. 118-30.

21. He D, Chen Y. Biogeography and molecular phylogeny of the genus Schizothorax (Teleostei: Cyprinidae) in China inferred from cytochrome b sequences. J Biogeogr. 2006;33:1448-60.

22. Liang $Y$, He D, Jia Y, Sun H, Chen Y. Phylogeographic studies of schizothoracine fishes on the Central Qinghai-Tibet plateau reveal the highest known glacial microrefugia. Sci Rep. 2017;7:10983.

23. Tao J, He D, Kennard MJ, Ding C, Bunn SE, Liu C, Jia Y, Che R, Chen Y. Strong evidence for changing fish reproductive phenology under climate warming on the Tibetan plateau. Glob Chang Biol. 2018;24:2093-104. 
24. Feng $X$, Jia $Y$, Zhu R, Chen K, Chen Y. Characterization and analysis of the transcriptome in Gymnocypris selincuoensis on the Qinghai-Tibetan plateau using single-molecule long-read sequencing and RNA-seq. DNA Res. 2019; 26:353-63.

25. Haas BJ, Papanicolaou A, Yassour M, Grabherr M, Blood PD, Bowden J, Couger MB, Eccles D, Li B, Lieber $M$, et al. De novo transcript sequence reconstruction from RNA-seq using the trinity platform for reference generation and analysis. Nat Protoc. 2013;8:1494-512.

26. Fu L, Niu B, Zhu Z, Wu S, Li W. CD-HIT: accelerated for clustering the nextgeneration sequencing data. Bioinformatics. 2012;28:3150-2.

27. Qi D, Chao Y, Wu R, Xia M, Chen Q, Zheng Z. Transcriptome analysis provides insights into the adaptive responses to hypoxia of a schizothoracine fish (Gymnocypris eckloni). Front Physiol. 2018;9:1326.

28. Li S. The scale formation process and its relation to the growth of five species of fish. J Fish China. 1983;7:343-51.

29. Sire JY, Arnulf I. Structure and development of the ctenial spines on the scales of a teleost fish, the cichlid Cichlasoma nigrofasciatum. Acta Zool. 2000;81:139-58.

30. White DS. Early development and pattern of scale formation in the spotted sucker, Minytrema melanops (Catostomidae). Copeia. 1977;1977:400-3.

31. Yu X, Li Y, Zhou T. Karyotype studies of cyprinid fishes in China: comparative study of the karyotypes of 8 species of schizothoracine fishes. J Wuhan Univ. 1990;02:97-104 (In Chinese).

32. Liu HP, Xiao SJ, Wu N, Wang D, Liu YC, Zhou CW, Liu Q, Yang R, Jiang WK, Liang $Q Q$, et al. The sequence and de novo assembly of Oxygymnocypris stewartii genome. Sci Data. 2019;6:190009.

33. Shimizu J, Asami N, Kataoka A, Sugihara F, Inoue N, Kimira Y, Wada M, Mano $H$. Oral collagen-derived dipeptides, prolyl-hydroxyproline and hydroxyprolyl-glycine, ameliorate skin barrier dysfunction and alter gene expression profiles in the skin. Biochem Bioph Res Co. 2015;456:626-30.

34. Montfort J, Le Cam A, Gabillard JC, Rescan PY. Gene expression profiling of trout regenerating muscle reveals common transcriptional signatures with hyperplastic growth zones of the post-embryonic myotome. BMC Genomics. 2016;17:810.

35. Li RW, Schroeder SG. Cytoskeleton remodeling and alterations in smooth muscle contractility in the bovine jejunum during nematode infection. Funct Integr Genomic. 2012;12:35-44.

36. Cao Y, Yao Z, Sarkar D, Lawrence M, Sanchez GJ, Parker MH, MacQuarrie KL, Davison J, Morgan MT, Ruzzo WL, et al. Genome-wide MyoD binding in skeletal muscle cells: a potential for broad cellular reprogramming. Dev Cell. 2010;18:662-74

37. Zhang J, Cui X, Shen Y, Pang L, Zhang A, Fu Z, Chen J, Guo X, Gan W, Ji C. Distinct expression profiles of LncRNAs between brown adipose tissue and skeletal muscle. Biochem Bioph Res Co. 2014;443:1028-34.

38. Aubin JE, Liu F, Malaval L, Gupta AK. Osteoblast and chondroblast differentiation. Bone. 1995:17:S77-83.

39. Knopf F, Hammond C, Chekuru A, Kurth T, Hans S, Weber CW, Mahatma G, Fisher S, Brand M, Schulte-Merker S, et al. Bone regenerates via dedifferentiation of osteoblasts in the zebrafish fin. Dev Cell. 2011;20:713-24.

40. Nishimoto SK, Waite JH, Nishimoto M, Kriwacki RW. Structure, activity, and distribution of fish osteocalcin. J Biol Chem. 2003;278:11843-8.

41. Sire JY, Allizard F, Babiar O, Bourguignon J, Quilhac A. Scale development in zebrafish (Danio rerio). J Anat. 1997;190:545-61.

42. Metz JR, De Vrieze E, Lock EJ, Schulten IE, Flik G. Elasmoid scales of fishes as model in biomedical bone research. J Appl Ichthyol. 2012;28:382-7.

43. Feng $X$, He D, Shan G, Tao J, Chen Y. Integrated analysis of mRNA and miRNA expression profiles in Ptychobarbus dipogon and Schizothorax oconnori, insight into genetic mechanisms of high altitude adaptation in the schizothoracine fishes. Gene Rep. 2017;9:74-80.

44. Chen S, Zhou Y, Chen Y, Gu J. Fastp: an ultra-fast all-in-one FASTQ preprocessor. Bioinformatics. 2018;34:1884-90.

45. Camacho C, Coulouris G, Avagyan V, Ma N, Papadopoulos J, Bealer K, Madden TL. BLAST+: architecture and applications. BMC Bioinformatics. 2009;10:421.

46. Buchfink B, Xie C, Huson DH. Fast and sensitive protein alignment using DIAMOND. Nat Methods. 2015;12:59-60.

47. Kanehisa M, Goto S. KEGG: Kyoto encyclopedia of genes and genomes. Nucleic Acids Res. 2000;28:27-30

48. Conesa A, Götz S, García-Gómez JM, Terol J, Talón M, Robles M. Blast2GO: a universal tool for annotation, visualization and analysis in functional genomics research. Bioinformatics. 2005;21:3674-6.

49. Langmead B, Salzberg SL. Fast gapped-read alignment with bowtie 2. Nat Methods. 2012;9:357-9.
50. Li B, Dewey CN. RSEM: accurate transcript quantification from RNA-Seq data with or without a reference genome. BMC Bioinformatics. 2011;12:323.

51. Robinson MD, McCarthy DJ, Smyth GK, Robinson MD, McCarthy DJ, Smyth GK. edgeR: a bioconductor package for differential expression analysis of digital gene expression data. Bioinformatics. 2010;26:139-40.

52. Livak KJ, Schmittgen TD. Analysis of relative gene expression data using real-time quantitative PCR and the $2^{-\Delta \Delta C T}$ method. Methods. 2001;25:402-8.

\section{Publisher's Note}

Springer Nature remains neutral with regard to jurisdictional claims in published maps and institutional affiliations.
Ready to submit your research? Choose BMC and benefit from:

- fast, convenient online submission

- thorough peer review by experienced researchers in your field

- rapid publication on acceptance

- support for research data, including large and complex data types

- gold Open Access which fosters wider collaboration and increased citations

- maximum visibility for your research: over $100 \mathrm{M}$ website views per year

At BMC, research is always in progress.

Learn more biomedcentral.com/submissions 